CLINICAL STUDY

\title{
A long-term follow-up study of mortality in transsexuals receiving treatment with cross-sex hormones
}

\author{
Henk Asscheman ${ }^{1}$, Erik J Giltay ${ }^{3}$, Jos A J Megens ${ }^{2}$, W (Pim) de Ronde ${ }^{1}$, Michael A A van Trotsenburg ${ }^{2}$ \\ and Louis J G Gooren ${ }^{1}$ \\ ${ }^{1}$ Endocrine Unit, Department of Internal Medicine and ${ }^{2}$ Center of Expertise on Gender Dysphoria, VU University Medical Center, PO Box 7057, NL-1007 \\ MB Amsterdam, The Netherlands and ${ }^{3}$ Department of Psychiatry, Leiden University Medical Center, NL-230O RC Leiden, The Netherlands
}

(Correspondence should be addressed to H Asscheman who is now at HAJAP, Valeriusstraat 4hs, $1071 \mathrm{MH}$ Amsterdam, The Netherlands; Email: h.ajap@worldonline.nl)

\begin{abstract}
Objective: Adverse effects of long-term cross-sex hormone administration to transsexuals are not well documented. We assessed mortality rates in transsexual subjects receiving long-term cross-sex hormones.

Design: A cohort study with a median follow-up of 18.5 years at a university gender clinic.

Methods: Mortality data and the standardized mortality rate were compared with the general population in 966 male-to-female (MtF) and 365 female-to-male (FtM) transsexuals, who started cross-sex hormones before July 1, 1997. Follow-up was at least 1 year. MtF transsexuals received treatment with different high-dose estrogen regimens and cyproterone acetate $100 \mathrm{mg} /$ day. FtM transsexuals received parenteral/oral testosterone esters or testosterone gel. After surgical sex reassignment, hormonal treatment was continued with lower doses.

Results: In the MtF group, total mortality was 51\% higher than in the general population, mainly from increased mortality rates due to suicide, acquired immunodeficiency syndrome, cardiovascular disease, drug abuse, and unknown cause. No increase was observed in total cancer mortality, but lung and hematological cancer mortality rates were elevated. Current, but not past ethinyl estradiol use was associated with an independent threefold increased risk of cardiovascular death. In FtM transsexuals, total mortality and cause-specific mortality were not significantly different from those of the general population.

Conclusions: The increased mortality in hormone-treated MtF transsexuals was mainly due to nonhormone-related causes, but ethinyl estradiol may increase the risk of cardiovascular death. In the FtM transsexuals, use of testosterone in doses used for hypogonadal men seemed safe.
\end{abstract}

European Journal of Endocrinology 164 635-642

\section{Introduction}

Psychological evaluation has shown that sex reassignment increases the well-being of transsexual subjects (1-3). Cross-sex hormone treatment has an important role in acquiring the secondary sex characteristics of the desired sex (4). Transsexuals often start taking sex hormones at young to middle age and in higher than recommended dosages. Fearing loss of secondary characteristics of the reassigned sex, transsexual subjects usually continue hormones lifelong. Previous reports from our clinic, in 1989 (5) and 1997 (6), assessed clinical endpoints, such as morbidity and mortality, in transsexuals receiving cross-sex hormones. Both these studies found no increase in mortality rates in subjects receiving cross-sex hormones compared with the general population, but reported higher than expected rates of completed suicide and death due to acquired immunodeficiency syndrome (AIDS) in maleto-female (MtF) transsexual subjects, while no increased morbidity/mortality was observed in female-to-male (FtM) transsexual subjects.

Several studies have looked at the effects of cross-sex hormone administration on laboratory variables related to cardiovascular risk in transsexuals, finding partially favorable and partially unfavorable effects (7-10). The skewed sex ratio in cardiovascular disease favoring women at all ages and the increasing incidence of cardiovascular disease after menopause were previously interpreted to indicate that estrogens are cardioprotective. By contrast, hyperandrogenemia in women is associated with increases in cardiovascular risk factors (11), which has led to the belief that androgens are detrimental to cardiovascular health (12). However, the large randomized trials (Heart and Estrogen/Progestin Replacement Study (13) and Women's Health Initiative 
(14)) refuted the cardioprotective effects of exogenous estrogens, in its generality, leading to revision of the practice of hormone replacement therapy in (post)menopausal women.

Another factor to be considered is the route of administration of estrogens, possibly having relevance for their adverse effects. Oral versus transdermal administration of $17 \beta$-estradiol $\left(\mathrm{E}_{2}\right)$ may impact differently on variables such as inflammation markers (15), lipoproteins (16), and coagulation markers (17). The pharmacological nature of the estrogen compound may be of significance too: oral administration of the synthetic compound ethinyl estradiol may have more negative effects on hemostasis than oral or transdermal $\mathrm{E}_{2}$ (18).

This study aims primarily to describe all-cause and cause-specific mortality rates in subjects receiving cross-sex hormone treatment. This analysis extends our previous reports by assessing the effects of longer term use of cross-sex hormones in subjects treated at this clinic, increasing the accrued person-years of follow-up data from 10152 (6) to 25 544. In addition, the effects of aging and co-morbidity have likely increased the number of endpoints, which will increase the impact and precision of the effect size measures with smaller confidence intervals (CIs); associations previously not detected due to the smaller sample size and lower power may now become apparent. We report the observed mortality rates in 1331 transsexuals followedup for a median period of more than 18 years, and we compare the observed number of deaths with the expected number as found in the general population. In a subanalysis, the type of estrogen (i.e. oral ethinyl estradiol versus other estrogen compound and routes of administration) is analyzed in relation to the risk of cardiovascular mortality.

\section{Subjects and methods}

Baseline and follow-up data of all transsexual subjects referred to our outpatient department since 1975 were entered into a cumulative database. In the present analysis on mortality aiming to measure longer term effects, we included only subjects who had started crosssex hormone treatment before July 1, 1997, followed-up for at least 1 year and included $2 \mathrm{MtF}$ who had died the first year of hormone administration.

In total, 1331 subjects met the above inclusion criteria, $966(72.6 \%) \mathrm{MtF}$ transsexuals, with a mean age of 31.4 years at the start of cross-sex hormones (range: 16-76 years), with 18678 patient-years of follow-up, and 365 (27.4\%) FtM transsexuals, with a mean age 26.1 years (range: $16-57$ years) at the start of hormone therapy with 6866 patient-years of follow-up. Subjects were followed-up until July 1, 2007, or until the date of death. In 2009, we could cross check our database against the National Civil Record Registry
(Gemeentelijke Basis Administratie) which registers all residents in the Netherlands and, if deceased, their date of death (but not cause of death). We identified another $45 \mathrm{MtF}$ and $3 \mathrm{FtM}$ subjects included in our database who had died before July 1, 2007, but were unknown to us in our initial analysis on mortality based on hospital records (19). Of these additional deaths, the cause of death could be ascertained in two out of three FtM $(66 \%)$, and in 27 out of $45(60 \%) \mathrm{MtF}$ transsexual subjects. The mean follow-up period of subjects receiving cross-sex hormones was $19.3 \pm 7.7$ years (median 18.6, range 0.7-44.5 years) in the MtF group. In the FtM group, the follow-up was $18.8 \pm 6.3$ years (median 18.4, range 4.7-42.6 years; Table 1 ).

The cause of death was ascertained by medical report or information from the family physician and was coded according to the International Classification of Disease (ICD-10, 10th revision 2007; www.who.int/ classification/icd10online). When initiating sex reassignment treatment, all subjects had agreed that their data could potentially be used in future scientific analysis with the provision that data could not be related to an individual person.

\section{Hormone treatment}

In MtF transsexuals, hormone treatment before sex reassignment surgery consisted of estrogens combined with anti-androgens. Until 1989, mainly ethinyl estradiol was prescribed in a dose of $100 \mu \mathrm{g} /$ day, and

Table 1 Baseline and treatment-related characteristics of 1331 male-to-female and female-to-male transsexuals who underwent cross-sex hormone treatment.

\begin{tabular}{lcc}
\hline & $\begin{array}{c}\text { Male-to-female } \\
\text { transsexuals }\end{array}$ & $\begin{array}{c}\text { Female-to-male } \\
\text { transsexuals }\end{array}$ \\
\hline$n$ & 966 & 365 \\
Age at start (mean \pm s.D.) & $31.4 \pm 11.4$ & $26.1 \pm 7.6$ \\
Range (years of age) & $16-76$ & $16-56$ \\
Age groups $(n(\%))$ & $329(34.1)$ & $204(55.9)$ \\
15-24 & $429(44.4)$ & $145(39.7)$ \\
$25-39$ & $199(20.6)$ & $16(4.4)$ \\
$40-64$ & $9(0.9)$ & 0 \\
65-80 & & \\
Smoking status $(n(\%))$ & $254(26.3)$ & $94(25.8)$ \\
Never & $373(38.6)$ & $131(35.9)$ \\
Current & $339(35.1)$ & $140(38.3)$ \\
Former or unknown & $619(64.2)$ & $197(54.0)$ \\
Starting date before 1990 & & \\
$(n(\%))$ & $834(86.7)$ & $343(94.0)$ \\
Sex reassignment & & \\
surgery $(n(\%))$ & $19.4 \pm 7.7$ & $18.8 \pm 6.3$ \\
Follow-up on hormone & & \\
treatment $($ years \pm s.D.) & $22(2.2)$ & $1(0.3)$ \\
$<5$ years $(n(\%))$ & $50(5.2)$ & $6(1.6)$ \\
$5-10$ years $(n(\%))$ & $229(23.7)$ & $111(30.4)$ \\
10-15 years $(n(\%))$ & $252(26.1)$ & $99(27.2)$ \\
$15-20$ years $(n(\%))$ & $190(19.7)$ & $86(23.5)$ \\
$20-25$ years $(n(\%))$ & $131(13.6)$ & $43(11.8)$ \\
25-30 years $(n(\%))$ & $92(9.5)$ & $19(5.2)$ \\
$>30$ years $(n(\%))$ & & \\
\hline
\end{tabular}


only small numbers of patients used estrogen injections or other oral estrogen compounds, such as conjugated estrogens. But after publication of an elevated risk of venous thrombosis associated with ethinyl estradiol use (5), particularly in patients over 40 years of age, we started to recommend transdermal $\mathrm{E}_{2}$ to all $\mathrm{MtF}$, particularly to those over 40 years of age. In those MtF who did not tolerate or refused transdermal estrogens, oral estradiol valerate $2-4 \mathrm{mg} /$ day was prescribed. However, some subjects were reluctant to change their previous estrogen therapy and continued with ethinyl estradiol.

Before surgical sex reassignment in MtF transsexuals (which includes orchiectomy), estrogens were always combined with anti-androgen treatment (usually cyproterone acetate $100 \mathrm{mg} /$ day and spironolactone 100-200 mg/day in < $<\%$ of MtF) to decrease testosterone levels and/or block androgen action. In the period before we started to advice not to use ethinyl estradiol, the standard practice was to reduce the dose of ethinyl estradiol to $50 \mu \mathrm{g} /$ day after surgery, or estrogen treatment was changed to transdermal or oral $E_{2}$. Furthermore, anti-androgens were discontinued, but about $30 \%$ of the MtF subjects experienced regrowth of undesired (facial) hair to some extent and asked for continuation of anti-androgens, though in significant lower doses.

FtM transsexuals were prescribed testosterone as esters intramuscularly $250 \mathrm{mg} / 2$ weeks, reduced postoperatively to every 3 weeks, oral testosterone undecanoate 160-240 mg/day (Andriol, not available in the USA) and, more recently, transdermal testosterone $50 \mathrm{mg} /$ day. If uterine bleeding persisted, a progestin was added until hysterectomy, usually lynestrenol.

It is of note that the Dutch health care system fully covers sex reassignment treatment, with the result that almost all transsexual subjects have undergone sex reassignment surgery 2 years after starting crosssex hormones. Consequently, the observed effects of sex hormones on biological systems in this study are largely attributable to exogenous hormones.

\section{Statistical analysis}

The observed number of deaths in the study population was set against the expected numbers of deaths (except from AIDS and drug abuse) derived from the 2001 mortality data of the general population provided by the Central Bureau of Statistics of the Netherlands (Centraal Bureau voor Statistiek on www.statline.cbs.nl) stratified per age group (i.e. 15-24; 25-39; 40-64; and 65-80 years of age) and biological sex. Numbers of deaths were adjusted for the years of follow-up on cross-sex hormone treatment. Expected number of deaths from AIDS and drug abuse, which varied largely from year to year, was calculated from specific reports by Statistics Netherlands. The risk was expressed as standardized mortality ratio (SMR), and the $95 \%$ CIs were calculated by regarding the observed number as a Poisson variable with tables based on Poisson distribution (20).

In a subanalysis, the association of use of ethinyl estradiol to mortality was analyzed. The use of ethinyl estradiol (dichotomized into i) never or former users during hormone administration, and ii) ongoing users) was analyzed in relation to all-cause mortality, cardiovascular mortality, mortality due to external causes, cancer mortality, and non-cardiovascular mortality in $964 \mathrm{MtF}$ transsexuals. The never/former users were combined into one reference group, as the risk of cardiovascular death was not increased in former versus never users of ethinyl estradiol. The potentially mediating or confounding variables such as age, smoking status, and starting date before 1990 were adjusted for Cox proportional hazards models by incrementally including them as covariates. The associations of different groups of ethinyl estradiol use and mortality were explored by selecting the first group (i.e. never or former users of ethinyl estradiol) as the reference category (i.e. a hazard ratio of 1 ). The software used was SPSS version 17.0 (SPSS, Inc., Chicago, IL, USA).

\section{Results}

\section{Baseline characteristics}

Baseline data and duration of follow-up in the patient groups are shown in Table 1. MtF transsexual subjects were older when they started cross-sex hormones $(31.4 \pm 11.4$ years) than FtM $(26.1 \pm 7.4$ years; $P<0.001)$. In the MtF group, 207 subjects $(21.4 \%)$ were over 40 years of age, and nine subjects $(0.9 \%)$ were even over 65 years of age, whereas only few FtM $(n=16,4.4 \%)$ were over 40 years of age at the start of cross-sex hormone treatment. The mean duration of follow-up was not significantly different between $\mathrm{MtF}$ and FtM subjects $(19.4 \pm 7.7$ vs $18.8 \pm 6.3$ years; $P=0.12$ ). The rate of sex reassignment surgery (defined as orchiectomy/penectomy + vaginoplasty in $\mathrm{MtF}$ and extirpation of the internal genitalia with both ovaries in FtM) was significantly lower in MtF compared to FtM subjects $(86.7$ vs $94.0 \%, P<0.001)$.

\section{Mortality rates in MtF transsexuals}

In the MtF group, 122 (12.6\%) out of 966 subjects had died during follow-up. When compared with the adjusted expected mortality in the general population, MtF had a significantly increased mortality with a SMR of 1.51 (95\% CI: 1.47-1.55; Table 2). The increased mortality in MtF in the 25-39 years of age group (SMR 4.47; 95\% CI: 4.04-4.92) was mainly due to the relatively high numbers of suicides (in six), drugs-related death (in four), and death due to AIDS (in 13 subjects).

In 40-64 year age group, the SMR of total mortality was increased with 1.42 (95\% CI: 1.35-1.48). 
Table 2 SMR adjusted for age and period of follow-up on hormone treatment by biological sex in 1331 male-to-female and female-to-male transsexual subjects.

\begin{tabular}{|c|c|c|c|c|}
\hline \multirow[b]{2}{*}{ Cause of death } & \multicolumn{2}{|c|}{ Male-to-female transsexuals } & \multicolumn{2}{|c|}{ Female-to-male transsexuals } \\
\hline & Observed cases & SMR $(95 \% \mathrm{Cl})$ & Observed cases & SMR $(95 \% \mathrm{Cl})$ \\
\hline Malignant neoplasm & 28 & $0.98(0.88-1.08)$ & 5 & $0.99(0.65-1.44)$ \\
\hline Lung & 13 & $1.35(1.14-1.58)$ & 1 & $1.06(0.26-3.19)$ \\
\hline Digestive tract & 3 & $0.42(0.28-0.60)$ & 2 & $2.41(0.90-5.18)$ \\
\hline Hematological & 6 & $2.58(1.97-3.30)$ & 1 & $2.86(0.69-8.57)$ \\
\hline Brain & 2 & $1.59(0.95-2.46)$ & 0 & - \\
\hline $\begin{array}{l}\text { Other: kidney, melanoma, bone, and } \\
\text { prostate in MtF. In FtM: leiomyosarcoma }\end{array}$ & 4 & $0.79(0.57-1.07)$ & 1 & $0.77(0.25-1.77)$ \\
\hline Ischemic heart disease & 18 & $1.64(1.43-1.87)$ & 1 & $1.19(0.39-2.74)$ \\
\hline Cerebrovascular accidents & 5 & $1.26(0.93-1.64)$ & 0 & - \\
\hline AIDS & 16 & $30.20(26.0-34.7)$ & 0 & - \\
\hline Endocrine/diabetes & 2 & $0.85(0.41-1.32)$ & 0 & - \\
\hline Respiratory system diseases & 4 & $0.85(0.61-1.14)$ & 0 & - \\
\hline Digestive system diseases & 3 & $1.01(0.68-1.45)$ & 1 & $2.56(0.62-7.69)$ \\
\hline Genitourinary system disease (ESRD) & 1 & $1.21(0.58-2.17)$ & 0 & - \\
\hline Nervous system disease (MS) & 0 & & 1 & $3.57(0.86-10.7)$ \\
\hline External causes & 24 & $7.67(6.84-8.56)$ & 2 & $2.22(1.07-5.44)$ \\
\hline Illicit drugs use & 5 & $13.20(9.70-17.6)$ & 1 & $25.00(6.00-32.5)$ \\
\hline Suicide & 17 & $5.70(4.93-6.54)$ & 1 & $2.22(0.53-6.18)$ \\
\hline Unknown/ill-defined symptoms & 21 & $4.00(3.52-4.51)$ & 2 & $2.08(0.69-4.79)$ \\
\hline Total & 122 & $1.51(1.47-1.55)$ & $1 \overline{2}$ & $1.12(0.89-1.59)$ \\
\hline
\end{tabular}

ESRD, end-stage renal disease; MS, multiple sclerosis

The higher rate as compared with the general population was largely explained by eight suicides (where only one was expected on the basis of mortality data in the general population) and 17 deaths from cardiovascular diseases (where only eight were expected). In the relatively small MtF group over 65 years of age, total mortality was not increased (SMR $0.95,95 \%$ CI: $0.86-1.06$ ) as compared to the general population.

In MtF, ischemic heart disease was the cause of death in 18 subjects (SMR 1.64; 95\% CI: 1.43-1.87). The mean age of occurrence of the lethal ischemic cardiac event was 59.7 years (range: $42-79$ years). The mean duration of estrogen use was 13.2 years (range: 2-42 years). Eleven of them $(61 \%)$ had been using ethinyl estradiol during a mean period of 9.7 years (range: 2-16 years), whereas the other seven had used transdermal estrogen $(n=2)$, stilbestrol $(n=1)$, tibolon $(n=1)$, or conjugated estrogens $(n=3)$ for a mean period of 16.9 years (range: 5-42 years). The mean age at the start of estrogen treatment was 45.9 years (range: 18-70 years), 46.5 years in ethinyl estradiol users, and 44.9 years in users of other estrogens. Nine $(50 \%)$ of the deceased subjects were current smokers, two non-smokers, and seven former smokers or unknown. Four $(22 \%)$ had hypercholesterolemia $(>6.5 \mathrm{mmol} / \mathrm{l}$ or $>250 \mathrm{mg} / \mathrm{dl})$. Four $(22 \%)$ had been diagnosed earlier with venous thrombosis, and five $(28 \%)$ had suffered a previous myocardial infarction.

Five MtF subjects died from stroke (SMR 1.26; 95\% CI: 0.93-1.64). Two subjects died before the age of 60, and the other three subjects died when they were 60 , 62 , and 75 years old; therefore, in 40-64 years of age, the SMR for fatal stroke was 2.11 (95\% CI: 1.32-3.21). All had been using ethinyl estradiol, and in only one of the two who had suffered a previous transient ischemic attack, the treatment regimen had been changed to transdermal estrogen.

In the Cox proportional hazard analysis of the type of estrogen treatment in MtF, current use of ethinyl estradiol was significantly associated with cardiovascular mortality, but not with an increased risk of all-cause mortality or mortality due to other causes. The threefold increased hazard ratio of cardiovascular mortality in current users compared with never and former users of ethinyl estradiol remained significant after adjustment for covariates (Table 3).

In the MtF group, the observed total number of deaths due to malignant neoplasm $(n=28)$ was not increased compared with the general population, but lung cancer $(n=13)$ showed a statistically significant increased SMR of 1.35 (95\% CI: $1.14-1.58)$. The risk of leukemia/ lymphoma with six deaths (one acute myeloid leukemia, one chronic lymphoid leukemia, one unclassified leukemia, and three non-Hodgkin lymphomas) was significantly increased with a SMR of 2.66 (95\% CI: 1.93-3.60).

External causes of death were increased almost eightfold due to suicide and illicit drug use. The suicide rate in MtF was increased sixfold. Thirteen out of the seventeen $(76 \%)$ had received psychiatric treatment in the past. No suicides occurred within the first 2 years of hormone treatment, while there were six suicides after 2-5 years, seven after 5-10 years, and four after more than 10 years of cross-sex hormone treatment at a mean age of 41.5 years (range 21-73 years). 
Table 3 Hazard ratios ( $95 \% \mathrm{Cls}$ ) of mortality according to the use of ethinyl estradiol in 964 male-to-female transsexuals during a median of 18.6 years of follow-up. Two deaths within the first year of follow-up were excluded to reduce the chance of reverse causation. Cardiovascular mortality was defined as death due to myocardial infarction or stroke.

\begin{tabular}{|c|c|c|c|}
\hline & \multicolumn{2}{|c|}{ Use of ethinyl estradiol } & \multirow[b]{2}{*}{$P$ value } \\
\hline & Never or former use & Continuous use & \\
\hline No. of male-to-female transsexuals & 596 & 368 & \\
\hline All-cause mortality & $69(11.6 \%)$ & $51(13.9 \%)$ & \\
\hline Crude & 1.00 & $1.13(0.78-1.62)$ & 0.53 \\
\hline Adjusted for age and smoking & 1.00 & $1.33(0.92-1.92)$ & 0.13 \\
\hline Fully adjusted $^{\mathrm{a}}$ & 1.00 & $1.28(0.88-1.86)$ & 0.20 \\
\hline Cardiovascular mortality & $8(1.3 \%)$ & $15(4.1 \%)$ & \\
\hline Crude & 1.00 & $2.82(1.19-6.65)$ & 0.02 \\
\hline Adjusted for age and smoking & 1.00 & $3.64(1.52-8.73)$ & 0.004 \\
\hline Fully adjusted ${ }^{\mathrm{a}}$ & 1.00 & $3.12(1.28-7.63)$ & 0.01 \\
\hline Mortality due to external causes ${ }^{b}$ & $12(2.0 \%)$ & $11(3.0 \%)$ & \\
\hline Crude & 1.00 & $1.40(0.62-3.17)$ & 0.43 \\
\hline Adjusted for age and smoking & 1.00 & $1.44(0.63-3.30)$ & 0.38 \\
\hline Fully adjusted $^{\mathrm{a}}$ & 1.00 & $1.36(0.60-3.10)$ & 0.46 \\
\hline Cancer mortality & $17(2.9 \%)$ & $11(3.0 \%)$ & \\
\hline Crude & 1.00 & $0.99(0.46-2.12)$ & 0.98 \\
\hline Adjusted for age and smoking & 1.00 & $1.24(0.57-2.67)$ & 0.59 \\
\hline Fully adjusted $^{\mathrm{a}}$ & 1.00 & $1.35(0.61-3.00)$ & 0.46 \\
\hline Non-cardiovascular mortality & $46(7.7 \%)$ & $30(8.2 \%)$ & \\
\hline Crude & 1.00 & $1.00(0.63-1.59)$ & 0.99 \\
\hline Adjusted for age and smoking & 1.00 & $1.16(0.73-1.84)$ & 0.54 \\
\hline Fully adjusted $^{\mathrm{a}}$ & 1.00 & $1.15(0.71-1.83)$ & 0.58 \\
\hline
\end{tabular}

$P$ values using Cox proportional hazards models.

${ }^{a}$ Adjusted for age, smoking status, and a starting date before 1990 (because before 1990, ethinyl estradiol was the standard estrogen prescribed).

DDeaths due to accidents, intentional self-harm and suicide, assault, drugs, and adverse effects.

Five (1.6\%) suicides were observed among the $304 \mathrm{MtF}$ who were still using cyproterone acetate and $12(1.8 \%)$ in the group of MtF no longer using cyproterone acetate. Six MtF subjects who committed suicide (35\%) had not undergone sex reassignment surgery because there had been doubts about their mental stability. In the whole group of MtF subjects, $87.6 \%$ underwent sex reassignment surgery.

Also death due to illicit drug use $(n=5)$ was relatively increased (SMR 13.2; 95\% CI: 9.7-17.6). All had been past or current substance abusers before the start of hormone treatment but had been evaluated as sufficiently mentally stable to undergo hormone treatment. Sixteen MtF transsexual subjects died from AIDS between 1986 and 2006 (SMR 30.2; 95\% CI: 26.0-34.7). The underlying cause of death could not be ascertained in $21(17.2 \%)$ of the 122 subjects who had died.

\section{Mortality rates in FtM transsexuals}

In the FtM group, 12 out of 365 (3.4\%) died during follow-up. When compared with the adjusted expected mortality in the general population, in FtM, the SMR of 1.12 (95\% CI: $0.87-1.42)$ was not significantly increased (Table 2). Compared with the MtF population, actual numbers were lower in the FtM group, which resulted in large CIs of the point estimates. The FtM group was also on average of younger age (only six over 65 years of age) compared to the MtF group. Only one myocardial infarction was observed in a 72-year-old FtM subject after 42 years of testosterone treatment. External causes of death were increased due to one death by illicit drug abuse, a cause of death extremely rare in the reference group of the female general population. Total number of cancer deaths was not different from the expected number. No deaths due to breast cancer was observed, and other cancer death categories were not statistically significantly different from those expected, but again this has to be set against larger CIs.

\section{Discussion}

In this large cohort with a median follow-up of more than 18 years, we observed in MtF transsexual subjects a $51 \%$ relatively increased mortality rate compared with the general male population, mainly due to increased rates of death from suicide, illicit drugs, AIDS, cardiovascular disease, and unknown causes. In FtM transsexuals, the observed mortality rate was not significantly increased compared with women in the general population. However, it should be taken into consideration that FtM started cross-sex hormones (testosterone) at a younger age than MtF (at mean age 26.1 years compared with MtF at age 31.1 years), and rarely started treatment after the age of 40 . The effects of aging may thus carry less weight in the FtM group (also smaller) than the MtF group. Follow-up of FtM in 
the 65-79 years of age group was only 35.4 patient years, implying that no firm conclusions can be drawn in this FtM age group nor in the FtM group as a whole.

The increased mortality risk in MtF in our cohort was characterized by a high SMR of suicide (of 5.70), AIDS (of 30.2), and illicit drug-related deaths (of 13.2). In our previous publication, the increased mortality rates due to suicide and AIDS had already been noted (6). Depressive mood changes have been reported in cyproterone acetate use but these are usually transient occurring during the first 6 months of use. No association of suicide with actual use of cyproterone acetate could be established. The main benefit of $50-100 \mathrm{mg} /$ day cyproterone acetate before surgery is the effective suppression of testosterone levels and counteracting the biological action of androgens, allowing the use of estrogens in a lower dosage and a more potent biological action, particularly on breast tissue. Psychological evaluation has shown that sex reassignment increases the well-being of transsexuals, but it should not be considered as a cure-all; it is rehabilitative relieving gender dysphoria, but some transsexual subjects may still experience other problems (e.g. comorbid psychiatric problems, social isolation, troubled relationships, prejudice, and discrimination).

Our present analysis, as compared to our earlier reports, comprises a larger study population and a longer follow-up, resulting in a more apparent increased mortality rate of cardiovascular disease in MtF. This may be partially explained by heavier smoking and a higher incidence of hypercholesterolemia in MtF than in the male general population. Moreover, long-term ethinyl estradiol use was independently associated with a threefold increased risk of cardiovascular death. Our findings in 1989 (5) of an increased incidence of venous thrombosis associated with the use of ethinyl estradiol had already led to a change in type of estrogen prescription for new patients starting cross-sex hormones, and nowadays only few MtF use ethinyl estradiol or other oral estrogens in high dose. The increased risk of cardiovascular mortality was observed only in those who were still using ethinyl estradiol. No increased risk was found in former users who had changed to other formulations and lower doses of estradiol. This is reassuring for those who have changed to other estrogen preparations. The increased risk with ethinyl estradiol can possibly be explained by the thrombogenic hemostatic changes: a large increase in APC resistance and a decrease in plasma protein $S$ that have been previously described by our group (13). The high prevalence of previous venous thrombosis (22\%) in those who died from cardiovascular causes supports this hypothesis. The favorable serum lipid changes associated with ethinyl estradiol (7) - an increase in highdensity lipoprotein cholesterol $(+20 \%)$ and a decrease in low-density lipoprotein cholesterol $(-12 \%)-$ did apparently not translate into a reduced risk of cardiovascular death. Recently, raised levels of circulatory inflammatory markers in transsexuals treated with high dose of oral estrogens have been reported, which could further contribute to the increased cardiovascular risk (10). An increased risk of cardiovascular disease was also reported in women using oral contraceptives (OC), in particular if they used OC pills with a higher ethinyl estradiol content $(50 \mu \mathrm{g})$, even more so when they were smokers (21-23). The increased risk, however, disappeared after discontinuing OC use (24). In those MtF who had continued using ethinyl estradiol, subjects had used equally relatively high doses of about $50 \mu \mathrm{g}$ /day up to advanced age, which could explain our finding of an increased rate of cardiovascular death.

The total cancer mortality rate was not increased. The statistically significant increase in mortality rate of lung cancer may be related to heavier smoking in the transsexual population. The increased mortality rate due to a variety of hematological cancers is puzzling. There are no reports of associations of hematological cancers with use of sex hormones. This may be a chance finding, or may be explained by the association of nonHodgkin lymphomas with HIV, the latter might have gone unreported. The decreased mortality rate for colon cancer, also observed in the Women's Health Initiative (14), is similarly remarkable, but also this needs confirmation in further studies. We did not observe any cases of breast cancer in the population studied, neither in MtF nor in FtM, in agreement with the low prevalence of breast cancer in the literature.

Our study has a number of limitations inherent in a cohort study. Firstly, it was not randomized nor placebocontrolled which would have been difficult, if not impossible given the nature of the study population. Comparing our cohort with the general population was probably the best available option for this research but it should be noted that this comparison is potentially biased and confounded by lifestyle factors, prone to associated pathology and other factors specific for the transsexual population besides cross-sex hormone treatment. Transsexual subjects, in particular MtF, differed in a number of regards with the general population. Before they presented themselves for sex reassignment, they have an increased history of suicide attempts, more psychopathology, and substance abuse, probably associated with the psychological burden of gender dysphoria, as well as an increased prevalence of HIV infection. Secondly, the data have been collected over a 30-year period, and follow-up was not entirely complete, $40 \%$ of the subjects had their last visit to the clinic before 2007. Our cross check with the Dutch civil registry in 2009 confirmed this assumption.

In summary, increased mortality in hormone-treated MtF transsexuals was mainly due to non-hormonerelated causes, such as suicide, AIDS, and drug abuse, but current use of ethinyl estradiol was associated with an increased risk of cardiovascular death. In FtM transsexuals, the use of testosterone in doses similar 
to those used for replacement for hypogonadal men seemed safe, but our data in over 65-year-old FtM were limited. In line with the Endocrine Society's Clinical practice guidelines on Endocrine Treatment of Transsexual Persons (25), we strongly recommend not to prescribe ethinyl estradiol (or OC, often selfadministered in higher dosages) to MtF transsexuals. Transdermal and low dose oral estradiol combined with anti-androgens are effective with fewer side effects in our experience and as published by others $(26,27)$. Consequently, since ethinyl estradiol is no longer used in our clinic since 2001, there is no indication to routinely test asymptomatic MtF before initiation of cross-sex therapy for (inherited) forms of thrombophilia (27), as long as the subject's history does not suggest any additional risk (25).

Lifestyle behaviors, which include healthy diets, smoking cessation, and regular exercise, may help to reduce cardiovascular risk especially in the group of MtF. Furthermore, intense preventive action may help reduce the mortality from suicide, AIDS, and drug abuse.

\section{Declaration of interest}

The authors declare that there is no conflict of interest that could be perceived as prejudicing the impartiality of the research reported.

\section{Funding}

This research did not receive any specific grant from a funding agency in the public, commercial, or not-for-profit sector.

\section{Acknowledgements}

We thank Prof. Paul Lips (Endocrine Unit) for facilitating the study, and all family physicians who helped gathering the data on causes of death.

\section{References}

1 Kuiper B \& Cohen-Kettenis P. Sex reassignment surgery: a study of 141 Dutch transsexuals. Archives of Sexual Behaviour 198817 439-457. (doi:10.1007/BF01542484)

2 Weyers S, Elaut E, De Sutter P, Gerris J, T'Sjoen G, Heylens G, De Cuypere G \& Verstraelen H. Long-term assessment of the physical, mental, and sexual health among transsexual women. Journal of Sexual Medicine 20096 752-760. (doi:10.1111/j.1743-6109. 2008.01082.x)

3 Murad MH, Elamin MB, Garcia MZ, Mullan RJ, Murad A, Erwin PJ \& Montori VM. Hormonal therapy and sex reassignment: a systemic review and meta-analysis of quality of life and psychosocial outcomes. Clinical Endocrinology 201072 214-231. (doi:10. $1111 / \mathrm{j} .1365-2265.2009 .03625 . \mathrm{x})$

4 Gooren LJ, Giltay EJ \& Bunck MC. Long-term treatment of transsexuals with cross-sex hormones: extensive personal experience. Journal of Clinical Endocrinology and Metabolism 2008 93 19-25. (doi:10.1210/jc.2007-1809)

5 Asscheman H, Gooren LJG \& Eklund PL. Mortality and morbidity in transsexual patients with cross-gender treatment. Metabolism 198938 869-873. (doi:10.1016/0026-0495(89)90233-3)
6 van Kesteren PJM, Asscheman H, Megens JAJ \& Gooren LJG. Mortality and morbidity in transsexual subjects treated with crosssex hormones. Clinical Endocrinology 199747 337-342. (doi:10. 1046/j.1365-2265.1997.2601068.x)

7 Elbers JM, Giltay EJ, Teerlink T, Scheffer PG, Asscheman H, Seidell JC \& Gooren LJ. Effects of sex steroids on components of the insulin resistance syndrome in transsexual subjects. Clinical Endocrinology $2003 \mathbf{5 8}$ 562-571. (doi:10.1046/j.1365-2265. 2003.01753.x)

8 Elbers JM, Asscheman H, Seidell JC \& Gooren LJ. Effects of sex steroid hormones on regional fat depots as assessed by magnetic resonance imaging in transsexuals. American Journal of Physiology 1999276 E317-E325.

9 Polderman KH, Gooren LJ, Asscheman H, Bakker A \& Heine RJ. Induction of insulin resistance by androgens and estrogens. Journal of Clinical Endocrinology and Metabolism 199479 265-271. (doi:10.1210/jc.79.1.265)

10 Giltay EJ, Lambert J, Gooren LJ, Elbers JM, Steyn M \& Stehouwer CD. Sex steroids, insulin, and arterial stiffness in women and men. Hypertension 199934 590-597.

11 Elting MW, Korsen TJ, Bezemer PD \& Schoemaker J. Prevalence of diabetes mellitus, hypertension and cardiac complaints in a followup study of a Dutch PCOS population. Human Reproduction 2001 16 556-560. (doi:10.1093/humrep/16.3.556)

12 Shaw LJ, Bairey Merz CN, Azziz R, Stanczyk FZ, Sopko G, Braunstein GD, Kelsey FZ, Kip KE, Cooper-DeHoff RM, Johnson BD, Vaccarino V, Reis SE, Bittner V, Hodgson K, Rogers W \& Pepine CJ. Postmenopausal women with a history of irregular menses and elevated androgen measurements at high risk for worsening cardiovascular event-free survival: results from the National Institute of Health- National Heart, Lung, and Blood Institute sponsored women's ischemia syndrome evaluation. Journal of Clinical Endocrinology and Metabolism 200893 1276-1284. (doi:10.1210/jc.2007-0425)

13 Hulley S, Grady D, Bush T, Furbert C, Herrington D, Riggs B \& Vittinghoff E. Randomized trial of estrogen plus progestin for secondary prevention of coronary heart disease in postmenopausal women Heart and Estrogen/progestin Replacement Study (HERS) Research Group. Journal of the American Medical Association 1998288 605-613. (doi:10.1001/jama.280.7.605)

14 Rossouw JE, Anderson GL, Prentice RL, LaCroix AZ, Koopersberg C, Stefanick ML, Jackson RD, Beresford SA, Howard BV, Johnson KC, Kotchen JM \& Ockene J. Risks and benefits of estrogen plus progestin in healthy postmenopausal women: principal results from the Women's Health Initiative randomized controlled trial. Journal of the American Medical Association 2002288 321-333. (doi:10.1001/jama.288.3.321)

15 Wilson R, Spiers A, Ewan J, Johnson P, Jenkins C \& Carr S. Effects of high dose oestrogen therapy on circulating inflammatory markers. Maturitas 200962 281-286. (doi:10.1016/j.maturitas. 2009.01.009)

16 Giltay EJ, Verhoef P, Gooren LJ, Geleijnse JM, Schouten EG \& Stehouwer CD. Oral and transdermal estrogens both lower plasma total homocysteine in male-to-female transsexuals. Atherosclerosis 2003168 139-146. (doi:10.1016/S0021-9150(03)00090-X)

17 Post MS, Christella M, Thomassen LG, van der Mooren MJ, Van Baal WM, Rosing J, Kenemans P \& Stehouwer CD. Effect of oral and transdermal estrogen replacement therapy on hemostatic variables associated with venous thrombosis: a randomized, placebo-controlled study in postmenopausal women. Arteriosclerosis, Thrombosis, and Vascular Biology 200323 1116-1121. (doi:10.1161/01.ATV.0000074146.36646.C8)

18 Toorians AWFT, Thomassen MCLGD, Zweegman S, Magdeleyns EJP, Tans G, Gooren LJG \& Rosing J. Venous thrombosis and changes of hemostatic variables during cross-sex hormone treatment in transsexual people. Journal of Clinical Endocrinology and Metabolism $2003 \mathbf{8 8}$ 5723-5729. (doi:10. 1210/jc.2003-030520)

19 Asscheman H, Giltay EJ, Megens JAJ \& Gooren LJG. Long term mortality in hormone-treated transsexuals. Program of the 91st Annual meeting of The Endocrine Society, Washington, DC, 2009, P3-314 (Abstract). 
20 Gardner MJ \& Altman DFG. Statistics with Confidence. London: British Medical Journal, 1989.

21 Khader YS, Rice J, John L \& Abueita O. Oral contraceptive use and the risk of myocardial infarction: a meta-analysis. Contraception 200368 11-17. (doi:10.1016/S0010-7824(03)00073-8)

22 Tanis BC, van den Bosch MA, Kemmeren JM, Cats VM, Helmerhorst FM, Algra A, van der Graaf Y \& Rosendaal FR. Oral contraceptives and the risk of myocardial infarction. New England Journal of Medicine 2001345 1787-1793. (doi:10.1056/ NEJMoa003216)

23 Shufelt CL \& Bairey Merz CN. Contraceptive hormone use and cardiovascular disease. Journal of the American College of Cardiology 200953 221-231. (doi:10.1016/j.jacc.2008.09.042)

24 Stampfer MJ, Willett WC, Colditz GA, Speizer FE \& Hennekens CH. A prospective study of past use of contraceptive agents and the risk of cardiovascular diseases. New England Journal of Medicine 1988 319 1313-1317. (doi:10.1056/NEJM198811173192004)

25 Hembree WC, Cohen-Kettenis P, Delemarre-van de Waal HA, Gooren LJ, Meyer WJ III, Spack NP, Tangpricha V \& Montori VM.
Endocrine treatment of transsexual persons: an Endocrine Society clinical practice guideline. Journal of Clinical Endocrinology and Metabolism 200994 3132-3154. (doi:10.1210/jc.20090345)

26 Dittrich R, Binder H, Cupisti S, Hoffman I, Beckmann MW \& Mueller A. Endocrine treatment of male-to-female transsexuals using gonadotropin-releasing hormone agonist. Experimental and Clinical Endocrinology \& Diabetes 2005113 586-592. (doi:10. 1055/s-2005-865900)

27 Ott J, Kaufmann U, Bentz EK, Huber JC \& Tempfer CB. Incidence of thrombophilia and venous thrombosis in transsexuals under cross-sex hormone therapy. Fertility and Sterility 201093 1267-1272. (doi:10.1016/j.fertnstert.2008.12.017)

Received 15 December 2010

Accepted 25 January 2011 